\section{Hiperplasia condilar, un enfoque actual del diagnóstico y tratamiento. Revisión de la literatura}

\section{Condylar hyperplasia, approach to diagnosis and treatment. Review of the literature}

\begin{abstract}
Resumen
La Hiperplasia condilar (HC) fue descrita inicialmente por Robert Adams en 1836. Esta patología mandibular presenta varios tipos que afectan el tamaño y la morfología ósea, alteran la oclusión y las estructuras dentolabiales, afectan indirectamente al maxilar superior, dando como resultado patrones faciales que se pueden caracterizar por: prognatismo mandibular, aumento unilateral del cóndilo, el cuello, la rama y el cuerpo, asimetría facial, disfunción de la articulación temporomandibular, desequilibrio neuromuscular y patología dolorosa miofacial. Esta condición de la $\mathrm{HC}$, hace que requiera de un tratamiento multidisciplinario, que según su severidad, compete a cirujanos bucomaxilofaciales, ortodoncistas, fisioterapeutas y cirujanos plásticos. El diagnóstico de la $\mathrm{HC}$ se basa en la anamnesis, el examen clínico y la evaluación imagenológica, consistente en radiografías panorámica cefalométrica, tomografía macizo facial (TEM-CBCT), gammagrafía ósea y en algunos casos estudio anátomo-patológico. El propósito de esta revisión es conocer de manera detallada el comportamiento y pronóstico de esta patología de acuerdo al tipo, con la finalidad de facilitar el diagnóstico y las opciones terapéuticas, según la severidad, la edad del paciente y la patología en su forma activa o inactiva.
\end{abstract}

Palabras clave: Asimetría facial; Cóndilo mandibular; Prognatismo; Anomalías maxilofaciales; Tomografía; Cirugía ortognática.

\begin{abstract}
Condylar hyperplasia (HC) was first described by Robert Adams in 1836. This mandibular pathology presents several types that affect bone size and morphology, alter occlusion and dentolabial structures, indirectly affect the maxilla, resulting in facial patterns that can be characterized by: mandibular prognathism, unilateral increase of the condyle, neck, branch and body, facial asymmetry, temporomandibular joint dysfunction, neuromuscular imbalance and myofacial pain pathology. This condition of the HC, requires a multidisciplinary treatment, which according to its severity, is the responsibility of oral and maxillofacial surgeons, orthodontists, physiotherapists, plastic surgeons. The diagnosis of $\mathrm{HC}$ is based on the anamnesis, the clinical examination and the imaging evaluation, consisting of panoramic cephalometric radiographs, macizo-facial tomography (TEM-
\end{abstract}

\section{Artículo de Revisión}

\author{
Gabriela Sedano Balbin 1,a, Fernando Pérez Vargas ${ }^{1, b}$ \\ Percy Romero Tapia ${ }^{2, c}$ \\ ${ }^{1}$ Universidad Nacional Mayor de San Marcos, Facultad \\ de Odontología, Lima, Perú. \\ ${ }^{2}$ Hospital Nacional Guillermo Almenara Irigoyen, Servicio \\ de Odontología, Lima, Perú. \\ a Cirujana Dentista. \\ ${ }^{b}$ Especialista en Ortodoncia y Ortopedia Maxilar. \\ c Especialista en Cirugía Buco Máxilofacial.

\section{Correspondencia:} \\ Percy Romero Tapia \\ Correo electrónico: \\ drpercyromero@gmail.com \\ Av. José Pardo 182- Dpto. 302. Miraflores. Lima, Perú.
}

\section{Coautores: \\ Gabriela Sedano Balbin \\ gsedano@posteswise.com \\ Fernando Pérez Vargas \\ Ifperezvargas@gmail.com}

\section{Editora:}

Sandra Patricia Palomino-Gómez

Universidad Nacional Mayor de San Marcos, Perú.

Conflicto de intereses: los autores declaran no tener conflictos de interés.

\section{Fuente de financiamiento: autofinanciado.}

Recibido: 23/09/18

Aceptado: 02/04/19

Publicado: 05/06/19 
CBCT), bone scintigraphy and in some cases anatomo-pathological study. The purpose of this review is to know in detail the behavior and prognosis of this pathology according to the type, in order to facilitate diagnosis and therapeutic options, according to the severity, the age of the patient and the pathology in its active form or inactive.

Keywords: Facial asymmetry; Mandibular condyle; Prognathism; Maxillofacial abnormalities; Tomography; Orthognathic surgery.

\section{Introducción}

La hiperplasia condilar (HC) es una alteración poco común que tiene la peculiaridad de un crecimiento óseo excesivo, siendo observada de forma bilateral o unilateral produciendo asimetría facial. Por lo general, este crecimiento excesivo produce alteraciones oclusales, funcionales y estéticas, acompañado de trastorno de la articulación temporomandibular en muchos de estos casos $^{1,2}$.

Es un trastorno del desarrollo que se debe a factores genéticos, traumáticos, funcionales, tumorales y hormonales; se manifiesta en la adolescencia, en un rango de edad entre los 10 y 30 años, sin embargo, hay casos que ocurren entre los 9 a 80 ańos de edad, esta discrepancia podría explicarse por el momento en que los pacientes buscan un tratamiento profesional ${ }^{1}$.

Existen diversos métodos diagnósticos que van desde fotografías extraorales, modelos de estudio estáticos y articulados, radiografía panorámica, radiografía posteroanterior, tomografía con reconstrucción $3 \mathrm{D}$, gammagrafías óseas y estudios histopatológicos para el diagnóstico de la hiperplasia ${ }^{3}$.

El tratamiento de la $\mathrm{HC}$ se basa en un enfoque multidisciplinario, con tratamientos ortodónticos quirúrgicos. Los tratamientos quirúrgicos observados con mayor frecuencia en la literatura son; condilectomía y cirugía ortognática. Se pueden usar como un método único o estar asociados ${ }^{4}$. También es importante eliminar el centro del crecimiento en casos en los que se demuestra la hiperplasia condilar activa, o, por el contrario, tratar la secuela con cirugía ortognática y/o compensación dentoalveolar ortodóntica cuando la patología está inactiva. Un adecuado diagnóstico de la HC requiere de la gammagrafía ósea de la articulación temporomandibular (ATM) que brinda importante información del estado de actividad del cóndilo ${ }^{2,3}$.

\section{Definición}

La HC es un crecimiento excesivo, progresivo y patológico del cóndilo mandibular, unilateral o bilateral ${ }^{5}$. La HC afecta la cabeza y cuello del cóndilo, el cuerpo y las ramas mandibulares. Es una enfermedad autolimitante y deformante, donde el crecimiento es desproporcionado al crecimiento fisiológico del individuo, y podría continuar hasta que se haya completado el desarrollo esquelético del adulto (crecimiento patológico) ${ }^{4,5}$. Por lo general es independiente del sexo y la raza, aunque se presenta más en mujeres ${ }^{6,7}$. Existen muchas etiologías sugeridas de $\mathrm{HC}$, que incluyen neoplasia, trauma, infec- ción, carga condilar anormal y factores de crecimiento aberrantes ${ }^{6}$.

\section{Epidemiología}

La HC puede ocurrir a cualquier edad y puede continuar más allá del período de crecimiento ${ }^{2}$. Raijmakers y cols. ${ }^{8}$, en el 2012, reportaron que mujeres con HC unilateral superan significativamente en número a los varones en las poblaciones de estudio con un 64\% (IC $95 \%, 58-70 \%, \mathrm{n}=275$ pacientes).

La HC tiene mayor predilección de forma unilateral y se ha reportado una significativa predisposición de las mujeres comparado con varones (razón 2:1), con una prevalencia para el cóndilo del lado derecho de $57 \%$ respecto al cóndilo del lado izquierdo de $43 \%{ }^{3,8}$.

Esta predilección femenina está relacionada con las diferencias hormonales, especialmente de estrógenos. Estas hormonas son mediadores de crecimiento de tejido óseo, y se expresan en el cartílago articular y las placas de crecimiento. Se ha encontrado evidencia experimental de síntesis local de estrógenos en la articulación temporomandibular ${ }^{2,3,8}$.

\section{Etiología}

Actualmente, los estudios científicos no reportan una etiología exacta para la HC, sin embargo, entre sus causas destacan distorsiones endocrinas (p. ej., factores de crecimiento similares a la insulina $[\mathrm{IGF}]^{2}$, malformaciones congénitas, microsomía hemifacial, atrofia hemifacial), factores ambientales (trauma prenatal y postnatal, infecciones, deficiencias en el aporte sanguíneo e hipervascularización, así como trastornos neurotróficos), factores funcionales (interferencias oclusales y hábitos), factores hormonales (problemas endocrinos y factores de crecimiento), factores neoplásicos (osteoma, osteocondroma y condroma), factores genéticos, traumatismo y artrosis ${ }^{3}$.

Öberg y cols. ${ }^{9}$ informaron que el aumento de la vascularización en el cóndilo puede causar un aumento en la actividad de crecimiento. Yang y cols. ${ }^{10}$ reportaron que la $\mathrm{HC}$ puede ser de origen genético y mostrar una transición autosómica dominante o relacionada con el cromosoma Y.

\section{Histopatología}

La histología típica de los tejidos blandos del cóndilo mandibular incluye cuatro capas: capa articular fibrosa, capa mesenquimal indiferenciada, capa transicional y capa de cartílago hipertrófica, con presencia de islas de condrocitos en el hueso trabecular. Se ha determinado 
que la HC activa muestra una capa mesenquimal más amplia que la del cóndilo normal ${ }^{2}$.

Si corresponde a un estado activo, se observa tres parámetros básicos: 1. El grosor total de la tapa del cartílago, 2. El espesor de la capa de células precondroblásticas, 3. La presencia de islas de cartílago en la capa ósea, considerada como frecuente en la HC.

\section{Clasificación de la hiperplasia condilar}

El crecimiento patológico de la HC puede acontecer en distintas zonas de la mandíbula. El crecimiento puede ser el resultado de un cóndilo agrandado, un cuello condilar alargado, o la inclinación hacia afuera o hacia abajo del cuerpo y la rama ${ }^{2}$. Debido a las variaciones en las ubicaciones de crecimiento excesivo, se han desarrollado varios sistemas de clasificación para caracterizar mejor la patología; así como también, varias terminologías para describir el crecimiento mandibular ${ }^{7}$.

Clasificación de la HC según Obwegeser y Makek ${ }^{11}$. Desarrollaron un sistema de clasificación basado en la asimetría y el vector de crecimiento predominante. Clasificaron tres tipos de hipertrofia condilar:

El tipo 1: llamado elongación hemimandibular, tiene un crecimiento exagerado en el vector horizontal. Se observa la desviación del mentón hacia el lado no afectado, sin una asimetría vertical correspondiente. La línea media mandibular también se desplaza hacia el lado contralateral. Como consecuencia, los molares mandibulares contralaterales a menudo se desvían lingualmente para permanecer en una oclusión adecuada con los molares maxilares. Si los molares contralaterales no pueden adaptarse al crecimiento, puede desarrollarse una mordida cruzada. Normalmente, el cóndilo no se ve afectado, pero el cuello suele ser deforme y delgado. La rama es alargada, que es la base para referirse al tipo 1 como elongación hemimandibular (Figura 1).
El tipo 2: se define como hiperplasia hemimandibular, está asociado a un crecimiento exagerado del vector vertical, y se caracteriza por una desviación mínima del mentón. Debido al excesivo crecimiento hacia abajo de la mandíbula, los molares superiores en el lado afectado compensan siguiendo el crecimiento hacia abajo de la mandíbula. El hueso alveolar maxilar en el lado ipsilateral crece excesivamente para mantener la oclusión. Si los molares maxilares no pueden seguir el crecimiento descendente excesivo, se produce una mordida abierta en el lado afectado. En el tipo $2 \mathrm{HC}$, frecuentemente el cóndilo aparece agrandado, y la cabeza suele ser irregular o deforme. También se ha informado que el cuello del cóndilo está engrosado y/o alargado (Figura 1).

El tipo 3: es una combinación de los tipos 1 y 2.

Clasificación de la HC según Wolford y cols. ${ }^{6}$ Desarrollaron un sistema de clasificación según las patologías que causan HC. Se clasifica en cuatro categorías basadas en características clínicas, de imagen, de crecimiento e histológicas. Estos son:

El tipo 1: se observa un crecimiento acelerado y prolongado que causa el alargamiento condilar y mandibular. Inicia en la adolescencia y se intensifica durante la fase de crecimiento puberal. La HC tipo 1 se divide en tipo $1 \mathrm{~A}$ y $1 \mathrm{~B}$. El tipo 1A se define como la elongación mandibular que se produce bilateralmente, mientras que el tipo $1 \mathrm{~B}$ se produce unilateralmente (HCU). El tratamiento de HC tipo 1 depende del crecimiento si está activo o detenido. Los pacientes entre los 20 y más años de edad no tendrán mayor crecimiento mandibular, y solo basta con procedimientos quirúrgicos ortognáticos para corregir la deformidad dentofacial y maloclusión. Si el paciente todavía es un adolescente, el proceso de crecimiento puede ser continuo y requerirá de tratamiento ortodóntico ortopédico prolongado, además de la posibilidad de cirugía ortognática ${ }^{6}$.
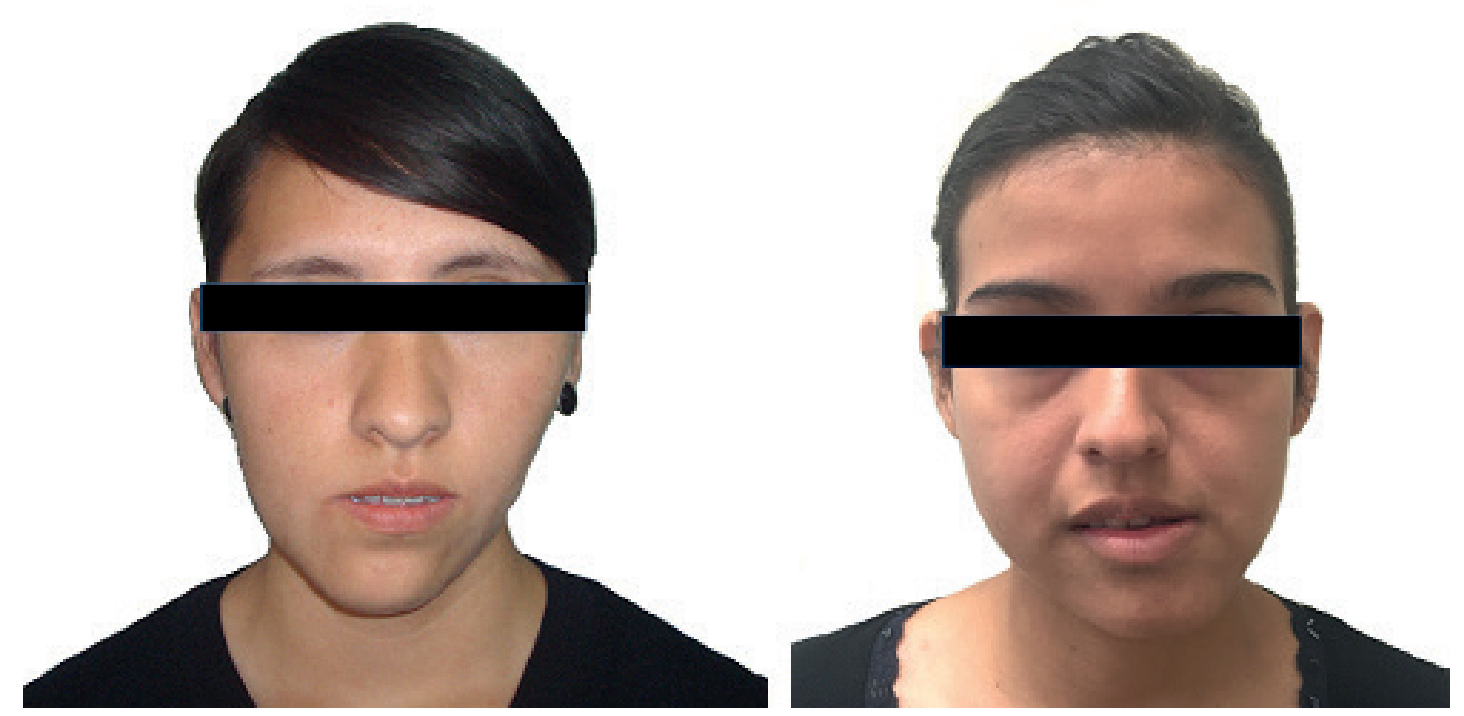

Figura 1. Pacientes con hiperplasia condilar unilateral donde se evidencia marcada deformidad dentofacial, con laterodesviación mandibular y asimetría facial 
Según el protocolo quirúrgico de Wolford ${ }^{6}$ existen dos opciones de tratamiento predecibles para el crecimiento activo del tipo 1: opción 1; a) realizar una condilectomía alta que elimina 4 a $5 \mathrm{~mm}$ la cabeza condilar del lado afectado (ambos lados para HC bilateral), b) reposicionar los discos articulares usando la técnica de anclaje Mitek; c) proseguir con los procedimientos quirúrgicos ortognáticos más adecuados, que consistirían en tratamiento ortodóntico y de cirugía ortognática.

La opción 2; consiste en realizar la cirugía ortognática, cuando se completa el crecimiento. Se considera que mientras más tiempo pase, peor será la deformidad facial, la asimetría y las compensaciones dentales, lo que afectará el desarrollo dentoesquelético y producirá un exceso de los tejidos blandos. Esto aumenta las dificultades para obtener un resultado funcional y estético óptimo, además de los efectos adversos sobre la oclusión, las compensaciones dentales, la masticación, el habla y el desarrollo psicosocial ${ }^{6,7}$.

El protocolo de tratamiento para la $\mathrm{HC}$ inactiva tipo $1 \mathrm{~B}$ son similares a las del tipo $1 \mathrm{~A}$ y por lo general se trata con ortodoncia y cirugía ortognática. La ATM debe abordarse solo si los discos articulares están desplazados. Si se confirma el crecimiento activo, entonces hay dos opciones de tratamiento ${ }^{6}$.

Opción 1. El protocolo quirúrgico de Wolford ${ }^{6}$ para el activo de tipo $1 \mathrm{~B}$ consiste en; a) realizar condilectomía alta unilateral para detener el crecimiento condilar aberrante; b) reposicionamiento de disco; c) procedimientos quirúrgicos ortognáticos, para optimizar los resultados funcionales y estéticos. Este protocolo quirúrgico es altamente predecible y estable, con una función normal de la mandíbula y una buena estética. En la opción 2: la cirugía se demora hasta que se completa el crecimiento óseo. Aproximadamente entre los 20 años, y luego solo se realiza la cirugía ortognática (Figura 2).

La cirugía para los casos de HC tipo 1B está indicada en pacientes no mayores de 15 años de edad para las niñas y de 17 a los 18 años para los niños. Sin embargo, la gravedad de la deformidad puede justificar la cirugía a una edad más temprana ${ }^{6}$.

La HC tipo 2 consiste en un desmesurado crecimiento unilateral del cóndilo causado por un osteocondroma y produce un sobrecrecimiento vertical de la mandíbula. La tasa de crecimiento varía de lenta a moderada, pero en algunos casos puede tener una tasa de crecimiento más rápida. Los osteocondromas son tumores benignos óseos más comunes, que representan el 35 al 50\% de todos los tumores benignos y del 8 al $15 \%$ de todos los tumores óseos primarios, y el tumor más común del cóndilo mandibular ${ }^{7-9}$.

El tratamiento incluye dos opciones. ${ }^{6}$ La opción 1; el protocolo quirúrgico de Wolford para el tipo 2 incluye a) una condilectomía, las técnicas de reconstrucción del cóndilo pueden incluir prótesis total de articulación temporomandibular, osteotomía de rama deslizante, injerto costal, injerto esternoclavicular, injerto óseo libre u ósea pediculada injerto. Cuando solo se aborda el cóndi- lo ipsilateral, sin cirugía ortognática adicional incluida, el paciente a menudo queda con resultados funcionales y estéticos no tan satisfactorios. Algunos de estos pacientes pueden requerir procedimientos ortognáticos secundarios para lograr una oclusión funcional y restaurar el buen equilibrio facial y la estética. Se clasifica en dos tipos adicionales, los tipos $2 \mathrm{~A}$ y $\mathrm{B}$. El tipo $2 \mathrm{~A}$ resulta del alargamiento vertical de la cabeza y el cuello condíleo. El tipo 2B implica el crecimiento tumoral exofítico horizontal del cóndilo además del alargamiento vertical de la cabeza y el cuello ${ }^{6}$.

El tipo 3 consiste en otros tumores benignos que causan $\mathrm{HC}$, incluidos, entre otros, osteomas, neurofibromas y displasia fibrosa, y da como resultado un agrandamiento facial unilateral ${ }^{6}$. El tipo 4 es causado por tumores malignos que se originan en el cóndilo y causan agrandamiento y asimetría facial. Algunos tumores malignos atribuidos a tipo 4 incluyen condrosarcoma, mieloma múltiple, osteosarcoma y sarcoma de Ewing ${ }^{6}$.

El tratamiento de la $\mathrm{HC}$ tipo 3 y 4 dependerá de la naturaleza y el alcance de la patología (benigna o maligna), las estructuras involucradas, la edad del paciente y otras afecciones médicas presentes. El tratamiento puede incluir la extirpación del tumor o el proceso patológico, con el tratamiento adyuvante apropiado para la neoplasia específica ${ }^{6}$.

Clasificación de la HC según Slootweg y Müller ${ }^{12}$. En 1986 propusieron una clasificación histológica, donde analizaron la capa fibrosa articular, la capa mesenquimal indiferenciada, la capa de transición y la capa de cartílago hipertrófica y caracterizaron cada capa en función de los hallazgos histológicos. Según ello la HC presentó las siguientes características:

La HC tipo I mostró una amplia zona de proliferación celular con engrosamiento de cartílago hialino y presencia de islas de cartílago en trabeculas óseas. La HC tipo II presentó también una zona de proliferación celular, engrosamiento de cartílago hialino y predominio de diferenciación de áreas pobre y ricamente proliferadas con mayor infiltración ósea por islas de cartílago ${ }^{12}$.

La HC tipo III presentó las mismas características vistas en el tipo I y tipo II con mayor irregularidad de la disposición celular. La HC tipo IV, mostró escasa formación de formación de fibrocartilago e inactividad de cartílago hialino ${ }^{12}$.

\section{Diagnóstico de la Hiperplasia Condilar}

Los métodos radiológicos, tomografía computarizada y gammagráficos se usan comúnmente para el diagnóstico y el control de los aspectos macroscópicos, siendo esenciales para un correcto diagnóstico ${ }^{13}$.

En los exámenes imagenológicos el cóndilo suele ser alargado pero también engrosado en las tres direcciones del espacio ${ }^{13}$. Se observa alteración en forma y tamaño del cóndilo, rama y cuerpo mandibular, con desviación del mentón. También se observa exceso vertical de los procesos alveolares mandibulares y maxilares con descompensación oclusal ${ }^{14}$ (Figura 2). 
Es necesario un examen radiológico que muestre la elongación del cuello y la cabeza del cóndilo para un diagnóstico definitivo. Las radiografías: panorámica, cefalométrica lateral y postero-anterior, así como las mediciones lineales y angulares halladas en estas, proporcionan información importante para detectar alteraciones esqueléticas en posición, forma y tamaño y determinar que estructuras cráneo-faciales están involucradas ${ }^{14-16}$.

Tomografía computarizada por emisión de fotón único (SPECT): el escáner es una parte importante del diagnóstico. SPECT produce una imagen de tomografía ósea tomografía que puede ser más confiable que la exploración plana ${ }^{5}$. Informa un aumento en la unidad condilar logrado con deformidades a veces importantes de la cabeza. El cuello del cóndilo a menudo es alargado y ancho ${ }^{14}$ (Figura 2).

Gammagrafía ósea: es un método instantáneo de comparación de la actividad metabólica celular entre el cóndilo normal y anormal. Puede utilizarse para distinguir el lado normal del anormal y para proporcionar información sobre la estabilidad o actividad de la hiperplasia ${ }^{13}$. La gammagrafía marca la actividad de los osteoblastos que rigen la estructura ósea. En un diagnóstico de HC clínica y radiográfica, una gammagrafía positiva muestra la naturaleza activa de la enfermedad ${ }^{14}$. Para casos asimétricos, los datos indican que una diferencia de actividad de más del 10\% entre las regiones condilares izquierda y derecha, que se encuentra con la gammagrafía ósea, es sugestiva de $\mathrm{CH}^{5,17}$.

Tomografía por emisión de positrones (PET): usa un análogo de glucosa radiomarcado, 18F-2 fluoro-2-desoxiglucosa (FDG), como un marcador, solo o combinado con tomografía computarizada (PET/CT). El PET proporciona una mejor resolución espacial que una cámara gamma convencional ${ }^{5}$.
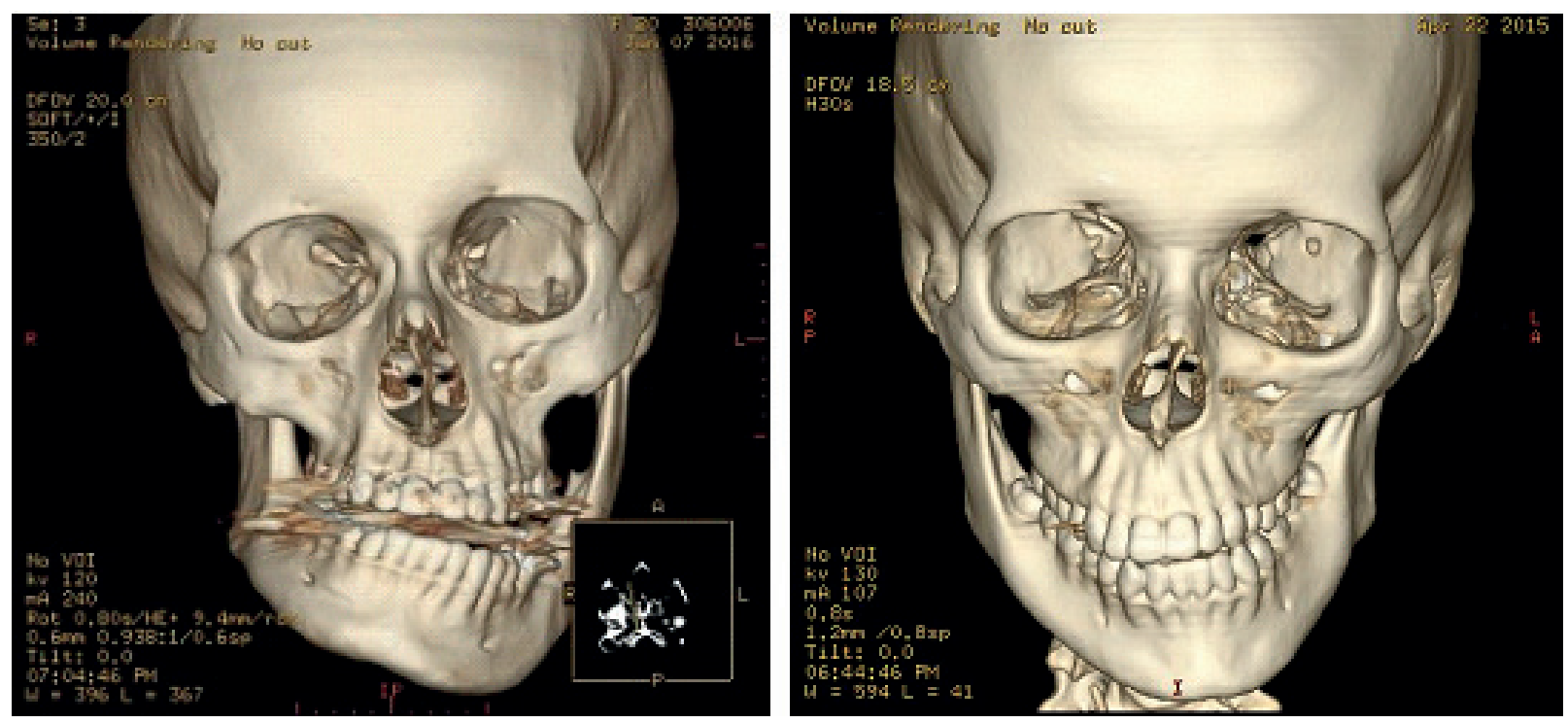

Figura 2. Vistas tomográficas en reconstrucción 3D de hiperplasias condilares unilaterales, tipo 2B (izquierda), tipo 1B (derecha) 
blandos. El diagnóstico diferencial se establece mediante la anamnesis, la evaluación clínica y los exámenes complementarios, de anormalidades malformativas congénitas con características similares (microsomía hemifacial, síndrome de Goldenhar, síndrome de Parry Romberg), o anomalías adquiridas (secuelas traumáticas o infecciosas, etc. $)^{14,15}$.

\section{Tratamiento de la hiperplasia condilar}

El tratamiento es multidisciplinario, pero principalmente ortodóntico-quirúrgico. Existe cierta controversia en cuanto a la opción de tratamiento ideal y el tiempo de tratamiento. Los planes de tratamiento deben considerar el grado de asimetría, la maloclusión resultante y la actividad de crecimiento condilar. Los tratamientos para corregir estos problemas se pueden abordar de forma conjunta o por separado. Por lo general, la estrategia seleccionada depende de la actividad de crecimiento y la edad del paciente. Como siempre, las demandas y expectativas del paciente son otras consideraciones importantes ${ }^{2,13,18,21}$.

Se han propuesto diferentes opciones quirúrgicas para tratar esta anomalía, que van desde la condilectomía alta (condilectomía conservadora) hasta la cirugía ortognática, o incluso una combinación de ambas. Por lo tanto, los pacientes con hiperplasia condilar activa tienen mejores resultados cuando se realiza una técnica de condilectomía alta ${ }^{19,22}$.

El tratamiento quirúrgico más complejo para la $\mathrm{HC}$ comprende condilectomía y/o cirugía ortognática. En un estudio realizado por Wolford y cols. ${ }^{20}$ el 2002, compararon los resultados del tratamiento de $\mathrm{HC}$ en un seguimiento a largo plazo de la estabilidad post-quirúrgica en pacientes diagnosticados con esta patología. Un grupo fue tratado solo con cirugía ortognática para co- rregir la asimetría, y el otro grupo fue tratado con condilectomía alta, reposicionamiento articular del disco y cirugía ortognática simultánea, demostrando que esta última es más estable y segura en el tiempo, con una diferencia estadísticamente significativa ${ }^{21}$ (Figura 3).

Para todas las opciones quirúrgicas, el tiempo, los resultados funcionales y estéticos, son esenciales para determinar el plan de tratamiento más adecuado para la HC. El tratamiento de ortodoncia antes y después del tratamiento quirúrgico es de mucha importancia para lograr una oclusión ideal ${ }^{22,23 .}$

La condilectomía alta del cóndilo afectado está indicada para limitar la progresión de la asimetría durante la fase activa del crecimiento excesivo condilar. Esta consiste en extraer la parte superior del cóndilo con una disección de 3-5 mm de la cabeza condilar, incluyendo los polos medial y lateral. Previamente, debe realizarse una disección profunda de la capa de la fascia y debe delimitarse el cóndilo del disco articular ${ }^{3}$.

La condilectomía debe hacerse una vez sea diagnosticada la HC, seguida de un tratamiento ortodóntico, ya sea de descompensación dentoalveolar, con el fin de preparar al paciente para una cirugía ortognática que corrija las secuelas de la enfermedad (asimetría), o de un tratamiento ortodóntico compensador, en casos en donde la enfermedad se haya detectado a tiempo y sea más un compromiso oclusal el que se deba corregir y no uno estético por alteración esquelética ${ }^{13}$. En los casos donde se sospeche la presencia de un tumor, este procedimiento es obligatorio ${ }^{18,19}$.

El tratamiento ortodóntico pre-quirúrgico de la $\mathrm{HC}$ en estado inactivo, es usado para alinear y nivelar los arcos dentales, para eliminar las compensaciones dentales producidas en los tres planos del espacio y para corre-

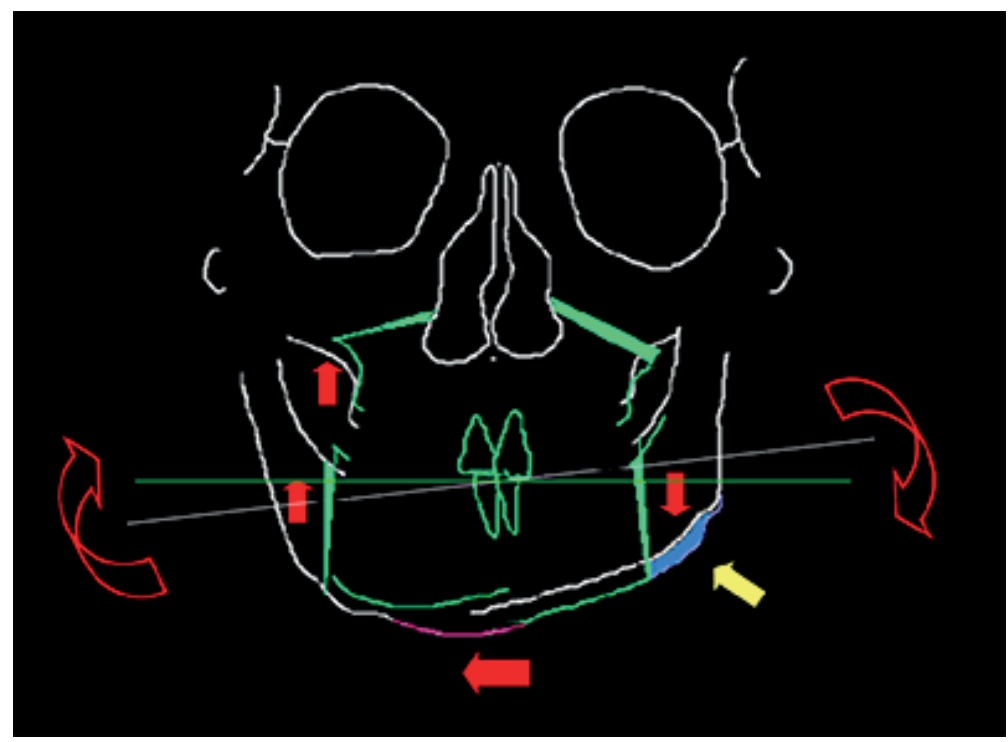

Figura 3. La HCU ocasiona descompensación dentoalveolar, la cual debe de ser corregida con compensación dentoalveolar ortodóntica más cirugía ortognática cuando la patología condilar está inactiva para corregir las secuelas funcionales y estéticas 
gir las inclinaciones, el torque y el eje axial de toda la dentición. Mientras que la ortodoncia post quirúrgica les entrega a los pacientes una oclusión ideal, funcional y estéticamente armónica con la nueva condición post quirúrgica del paciente ${ }^{24-28}$.

Recientemente, se ha introducido un nuevo procedimiento quirúrgico para la hiperplasia condilar denominado "primer acercamiento a la cirugía" (SFA: surgery first approach). Este procedimiento tiene como objetivo detener la HC, corregir la discrepancia esquelética y la maloclusión, a la vez que satisface los requisitos estéticos del paciente ${ }^{29}$.

La reducción del tiempo de tratamiento es posible debido a que el fenómeno de aceleración regional (RAP: regional acceleration phenomenon), permite que la descompensación dental se corrija de mejor forma con la cirugía ${ }^{30}$. El RAP es un proceso fisiológico complejo con características específicas que abarca la remodelación ósea rápida y la pérdida de densidad ósea regional. El RAP aumenta la reorganización del tejido y la curación mediante el aumento transitorio de la resorción ósea localizada y una mayor remodelación. Esta es la explicación de la aceleración del movimiento dental ortodóntico durante un período de 4 a 5 meses aproximadamente ${ }^{26,28-30}$.

El protocolo consiste en realizar condilectomía alta para detener el estado activo de la hiperplasia. Un mes después de la condilectomía se realiza una impactación ortognática de la mandíbula y una cirugía de retracción mandibular asimétrica con la SFA. Después de 10 días, se realizan controles de ortodoncia cada dos semanas durante 4 hasta 6 meses, fisioterapia orofacial, drenaje linfático, rehabilitación neuromuscular y recuperación neurosensorial, con la consecuente alta del paciente ${ }^{29}$.

\section{Conclusiones}

- La hiperplasia condilar es un crecimiento excesivo, progresivo y patológico del cóndilo mandibular, unilateral o bilateral que se presenta con mayor frecuencia en la etapa de desarrollo y crecimiento. Mayormente se desarrolla en el sexo femenino unilateralmente.

- El diagnóstico de la hiperplasia condilar requiere de evaluación minuciosa con exámenes auxiliares detallados. Estos exámenes y herramientas son las siguientes: anamnesis, examen clínico, fotografía clínica, modelos de estudio, la radiografía, la gammagrafía ósea, la tomografía computarizada (TEM, CBCT, SPECT), todos estos métodos utilizados también ayudan a planificar el tratamiento ortodóntico quirúrgico.

- El tratamiento con mejor resultado consiste en una terapia multidisciplinaria, que abarca tratamiento ortodóntico y procedimientos quirúrgicos como la condilectomía y la cirugía ortognática; con el objetivo de mejorar la relación intermaxilar, la funcionalidad masticatoria y la estética del paciente.
- La gammagrafía y la tomografía computarizada por emisión de fotón único SPECT, técnicas de imagen de la medicina nuclear convencional, son los mejores métodos para identificar a los pacientes con hiperplasia condilar uni o bicondilar, métodos adecuados no solo para diagnosticar, sino también para elegir la terapia más adecuada dependiendo de su presentación en sus formas activa o inactiva.

- El manejo correcto de conceptos actualizados es importante para un tratamiento exitoso. Esto requiere de una constante información en protocolos de tratamiento modernos basados en investigaciones científicas.

\section{Referencias bibliográficas}

1. Pinto I, Fonseca J, Vinagre A, Ângelo D, Sanz D, Grossmann E. Mandibular condylar hyperplasia: diagnosis and management. Case report. Rev Dor. São Paulo, 2016;17(4):307-11.

2. Almeida L, Zacharias J, Pierce S. Condylar hyperplasia: An updated review of the literature. Korean J Orthod. 2015;45(6):333-40.

3. López D, Corral C. Hiperplasia condilar: características, manifestaciones, diagnóstico y tratamiento. Revisión de tema. Rev Fac Odontol Univ Antioq 2015;26(2):425-46.

4. Elbaz J, Wiss A, Raoul G, Leroy X, Hossein-Foucher C, Ferri J. Condylar hyperplasia: correlation between clinical, radiological, scintigraphic, and histologic features. J Craniofac Surg. 2014;25(3):1085-90.

5. Rodrigues D, Castro V. Condylar hyperplasia of the temporomandibular joint: types, treatment, and surgical implications. Oral Maxillofac Surg Clin North Am. 2015;27(1):155-67.

6. Wolford L, Movahed R, Perez D. A classification system for conditions causing condylar hyperplasia. J Oral Maxillofac Surg. 2014;72(3):567-95.

7. Yılancı H, Akkaya N, Özbek M. Terminology and classification of condylar hyperplasia: Two case reports and review. Kulak Burun Bogaz Ihtis Derg. 2015;25(6):367-74.

8. Raijmakers P, Karssemakers L, Tuinzing D. Female predominance and effect of gender on unilateral condilar hyperplasia: a review and meta-analysis. J Oral Maxillofac Surg. 2012;70(1): e72-6.

9. Oberg T, Fajers C, Lysell G, Friberg U. Unilateral hyperplasia of the mandibular condylar process. A histological, microradiographic, and autoradiographic examination of one case. Acta Odontol Scand 1962;20:485-504.

10. Yang J, Lignelli J, Ruprecht A. Mirror image condilar hyperplasia in two siblings. Oral Surg Oral Med Oral Pathol Oral Radiol Endod 2004;97:281-5.

11. Obwegeser H, Makek M. Hemimandibular hyperplasia--hemimandibular elongation. J Maxillofac Surg 1986;14:183-208.

12. Slootweg P, Müller H. Condylar hyperplasia. A clinico-pathological analysis of 22 cases. J Maxillofac Surg 1986;14:209-14.

13. Portelli M, Gatto E, Matarese G, Militi A, Catalfamo L, Gherlone E, Lucchese A. Unilateral condylar hyperpla- 
sia: diagnosis, clinical aspects and operative treatment. A case report. Eur J Paediatr Dent. 2015;16(2):99-102.

14. Ferri J, Raoul G, Potier J, Nicot R. Temporomandibular joint (TMJ): Condyle hyperplasia and condylectomy. Rev Stomatol Chir Maxillofac Chir Orale. 2016;117(4):259-65.

15. Nolte J, Karssemakers L, Grootendorst D, Tuinzing D, Becking A. Panoramic imaging is not suitable for quantitative evaluation, classification, and follow up in unilateral condylar hyperplasia. Br J Oral Maxillofac Surg. 2015;53(5):446-50.

16. Bharathi S, Senthilnathan S, Kumar L, Mohan A, Taranath $\mathrm{M}$. Unilateral condylar hyperplasia: A case report and review of literature. J Int Soc Prev Community Dent. 2014;4(1):67-70.

17. Bing Wen, Ying Shen and Chang-Yin Wang. Clinical Value of 99Tcm-MDP SPECT Bone Scintigraphy in the Diagnosis of Unilateral Condylar Hyperplasia. Scientific World Journal. 2014; 2014: 256256. DOI: $10.1155 / 2014 / 256256$.

18. López B, Corral S C. Comparison of planar bone scintigraphy and single photon emission computed tomography for diagnosis of active condylar hyperplasia. J Craniomaxillofac Surg. 2016;44(1):70-4.

19. Pereira-Santos D, De Melo W, Souza FA, de Moura W, Cravinhos J. High condylectomy procedure: a valuable resource for surgical management of the mandibular condylar hyperplasia. J Craniofac Surg. 2013;24(4):1451-3.

20. Wolford L, Mehra P, Reiche-Fischel O, Morales- Ryan C, García-Morales P. Efficacy of high condylectomy for management of condilar hyperplasia. Am J Orthod Dentofacial Orthop 2002;121:136-50.

21. Singh V, Verma A, Attresh G, Batra J. Ortho-surgical management of condylar hyperplasia: Rare case reports. Natl J Maxillofac Surg. 2014;5(1):54-9.
22. Rajkumar G, Hemavathy M, Shashikala R. Conservative management of unilateral condylar hyperplasia. J Oral Maxillofac Surg. 2012;16:201-5.

23. Xavier S, Santos de S, Silva E, Faria A, de Mello Filho F. Two-stage treatment of facial asymmetry caused by unilateral condylar hyperplasia. Braz Dent J. 2014;25:257-60.

24. Ferreira S, Da Silva-Fabris A, Ferreira G. Unilateral condylar hyperplasia: a treatment strategy. J Craniofac Surg. 2014;25(3):e256-8.

25. Wolford L, Movahed R, Dhameja A3, Allen W. Low condylectomy and orthognathic surgery to treat mandibular condylar osteochondroma: a retrospective review of 37 cases. J Oral Maxillofac Surg. 2014;72(9):1704-28.

26. Fariña R, Pintor F, Pérez J, Pantoja R, Berner D. Low condylectomy as the sole treatment for active condylar hyperplasia: facial, occlusal and skeletal changes. An observational study. Int J Oral Maxillofac Surg. 2015;44(2):217-25.

27. Ghawsi S, Aagaard E, Thygesen T. High condylectomy for the treatment of mandibular condylar hyperplasia: a systematic review of the literature. Int J Oral Maxillofac Surg. 2016;45(1):60-71.

28. López D, Aristizábal J, Martínez-Smit R. Condylectomy and "surgery first" approach: An expedited treatment for condylar hyperplasia in a patient with facial asymmetry. Dental Press J Orthod. 2017;22(4):86-96.

29. Yu CC, Chen PH, Liou EJ, Huang CS, Chen YR. A Surgery-first approach in surgical-orthodontic treatment of mandibular prognathism--a case report. Chang Gung Med J. 2010 Nov-Dec;33(6):699-705.

30. Frost HM. The regional acceleratory phenomenon: A review. Henry Ford Hosp Med J. 1983;31:3-9. 